Article

\title{
Optimal Kinematic Design of a 6-UCU Kind Gough-Stewart Platform with a Guaranteed Given Accuracy
}

\author{
Guojun Liu \\ School of Mechanical Engineering, Hunan Institute of Science and Technology, Yueyang 414006, China; \\ liuguojun_iest@163.com; Tel.: +86-138-7309-3067
}

Received: 24 April 2018; Accepted: 15 June 2018; Published: 18 June 2018

\begin{abstract}
The 6-UCU (U-universal joint; C-cylinder joint) kind Gough-Stewart platform is extensively employed in motion simulators due to its high accuracy, large payload, and high-speed capability. However, because of the manufacturing and assembling errors, the real geometry may be different from the nominal one. In the design process of the high-accuracy Gough-Stewart platform, one needs to consider these errors. The purpose of this paper is to propose an optimal design method for the 6-UCU kind Gough-Stewart platform with a guaranteed given accuracy. Accuracy analysis of the 6-UCU kind Gough-Stewart platform is presented by considering the limb length errors and joint position errors. An optimal design method is proposed by using a multi-objective evolutionary algorithm, the non-dominated sorting genetic algorithm II (NSGA-II). A set of Pareto-optimal parameters was found by applying the proposed optimal design method. An engineering design case was studied to verify the effectiveness of the proposed method.
\end{abstract}

Keywords: accuracy analysis; optimal design; multi-objective evolutionary algorithms; NSGA-II; 6-UCU kind Gough-Stewart platform

\section{Introduction}

With the advantages of high rigidity, high precision, and large carrying capacity, Gough-Stewart platforms (GSPs) are extensively used in virtual-reality motion simulators [1]. Many scholars studied the optimal design of the 6-UPS kind GSP [1], where U stands for the universal joint, P for the prismatic joint, and $S$ for the spherical joint. When compared with spherical joints, universal joints can bear more tension [2]. Universal joints are extensively used as the passive joints of GSPs to connect hydraulic cylinders or electric cylinders to the moving platform and fixed base. Universal joints are used as the passive joints of the universal tyre test machine designed by Gough et al. [3-5], the motion simulator patented by Cappel [6], commercial flight simulators [7], the Ampelmann system [8], the Moog FCS 5000E motion base [9], the VARIAX machine tool [10], and the AMiBA hexapod telescope mount [11]. Universal joints are also used as the passive joints of the docking test system, and about 60 motion simulators designed by the team of Professor Junwei Han at the Harbin Institute of Technology, China. The cylinders of the hydraulic and electric actuators that can not only translate along the axis, but also rotate along the axis are cylindrical joints instead of prismatic ones. These GSPs are 6-UCU parallel manipulators [12,13], where C stands for the cylinder joint.

In highly accurate positioning applications, such as the docking test systems, high accuracy is required to achieve good simulation results. in China, the translational motion errors of the moving platform must be less than $1 \mathrm{~mm}$ in the whole workspace of the docking test system [14]. Many researchers studied the accuracy analysis of GSPs. Wang and Masory studied the effects of manufacturing errors on the accuracy of a GSP by modeling the GSP as serial legs based on the 
Denavit-Hartenberg method [15]. Ropponen and Arai presented the error model of a modified GSP by using the differentiation method [16]. Patel and Ehmann established an error model of a GSP, addressing all possible sources of errors [17]. Wang and Ehmann developed first- and second-order error models of a GSP [18]. Masory et al. presented an effective method of identifying kinematic parameters of a GSP using pose measurements [19]. Cong et al. developed a kinematic calibration method of a GSP by using a three-dimensional coordinate measuring machine containing actuator errors and passive joint errors [20]. Dai et al. proposed an accuracy analysis method for a docking test system [14]. Merlet and Daney proposed an optimal method of parallel manipulator considering manufacturing errors by using interval analysis [21-23]. Because of the dependence of the variables, a high level of expertise in interval analysis is needed for the proper usage of parallel manipulators [22]. To the best of our knowledge, there are few papers considering the errors of a GSP in optimal design processes.

In the optimal design process of a high-precision GSP, one needs to find the optimal solutions which meet the given accuracy requirement. An optimal design method for a GSP is proposed in this paper, so as to meet these accuracy requirements.

In this paper, the error model of the 6-UCU kind GSP is derived in Section 2. The optimal design method is proposed in Section 3. Section 4 presents a case study to illustrate the effectiveness of the proposed optimal design. The conclusions are given in Section 5 .

\section{Differential Error Model}

In this section, the relationship between the structural errors and end-point errors of a 6-UCU kind GSP is discussed. The 6-UCU kind GSP consisted of a moving platform, a fixed base, and six limbs, as shown in Figures 1 and 2. The $i$ th limb was connected to the fixed base via a universal joint, whose joint center point is denoted as $B_{i}$, and connected to the mobile platform via a universal joint with center $P_{i}$, as shown in Figure 2. For convenience, a Cartesian coordinate frame, $O_{1}-X_{1} Y_{1} Z_{1}$, was attached to the moving platform with origin $O_{1}$. A Cartesian coordinate frame, $O-X Y Z$, was attached to the fixed base with origin $O$.

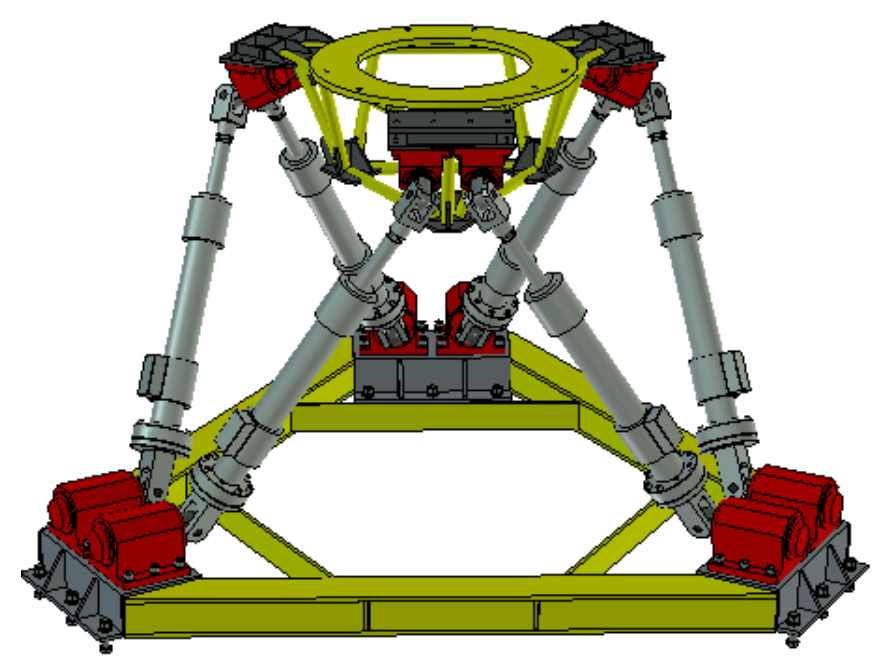

Figure 1. The 6-UCU (U—universal joint; C—cylinder joint) kind Gough-Stewart platform (GSP). 


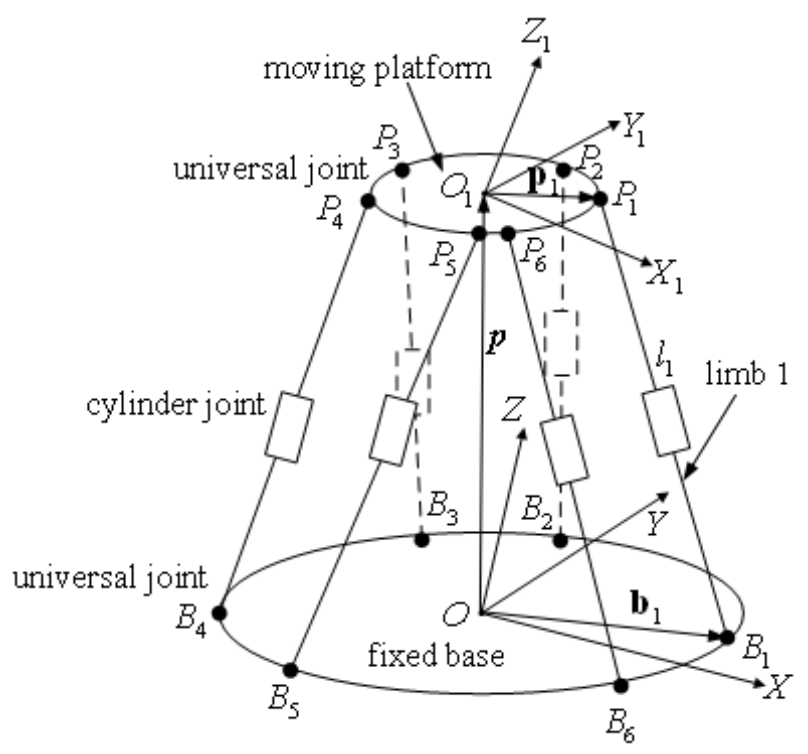

Figure 2. Schematic of the 6-UCU kind GSP.

The positions of the universal joints attached to the moving platform and the fixed base of the GSP formed semi-hexagons, as shown in Figure 3, where $r_{P}$ is the radius of the platform-joint attachment circle, $d_{P}$ is the distance between the shorter edges of the attachment point semi-hexagon on the moving platform, $r_{B}$ is the radius of the base-joint attachment circle, and $d_{B}$ is the distance between the shorter edges of the attachment point semi-hexagon on the fixed base.

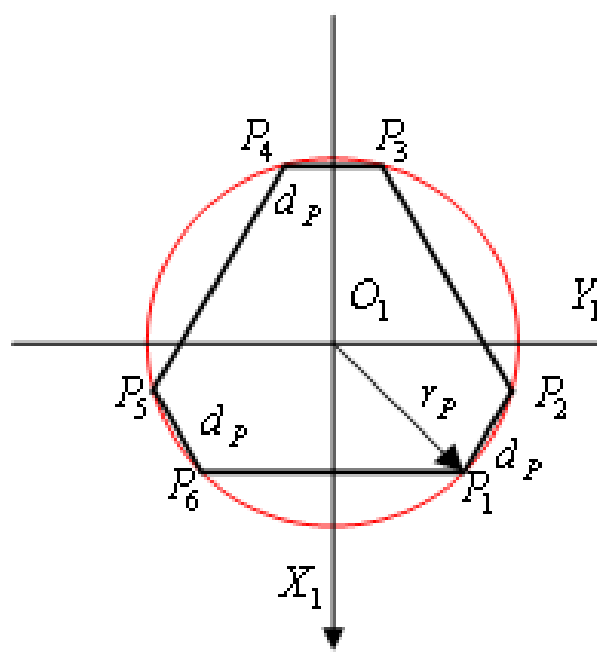

(a)

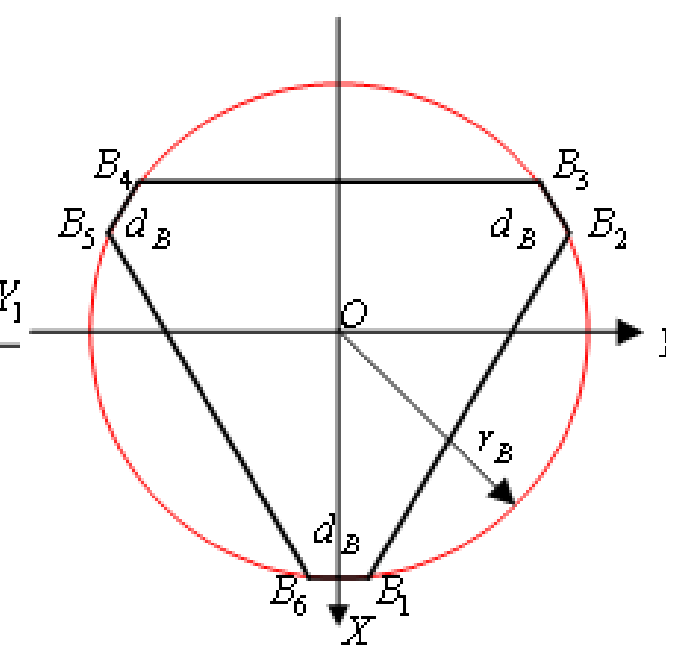

(b)

Figure 3. Schematic of the universal joint positions. (a) Moving platform; and (b) fixed base.

Referring to Figures 1 and 2, the position vector of $P_{i}$ expressed in the frame $O-X Y Z$ could be written as

$$
\mathbf{p}+\mathbf{R}^{\mathbf{L}} \mathbf{p}_{i}=\mathbf{b}_{i}+l_{1 i} \mathbf{n}_{1 i}+l_{2 i} \mathbf{n}_{2 i}
$$

where $\mathbf{R}$ is the rotation matrix from the frame $O_{1}-X_{1} Y_{1} Z_{1}$ to $O-X Y Z$, $\mathbf{p}$ is the position coordinate of the point $O_{1}$ measured in the frame $O-X Y Z, \mathbf{b}_{i}$ is the position coordinate of the point $B_{i}$ measured in the frame $O-X Y Z, \mathbf{p}_{i}$ is expressed as ${ }^{\mathbf{L}} \mathbf{p}_{i}$ in the frame $O-X Y Z,{ }^{\mathbf{L}} \mathbf{p}_{i}$ is the coordinate of the point $P_{i}$ measured in the frame $O_{1}-X_{1} Y_{1} Z_{1}, l_{1 i}$ is the distance from the point $B_{i}$ to the lower plane of the 
piston, $l_{2 i}$ is the distance from the point $P_{i}$ to the lower plane of the piston, $\mathbf{n}_{i}$ is the unit vector of $\overrightarrow{\mathrm{B}_{i} \mathrm{P}_{i}}$, $\mathbf{n}_{1 i}$ and $\mathbf{n}_{2 i}$ are the unit vectors of $\mathbf{n}_{i}$ fixed to the cylinder and piston, respectively, and $\mathbf{n}_{i}=\mathbf{n}_{1 i}=\mathbf{n}_{2 i}$.

${ }^{\mathbf{L}} \mathbf{p}_{i}$ and $\mathbf{b}_{i}$ are

$$
\begin{aligned}
{ }^{\mathbf{L}} \mathbf{p}_{i} & =\left[\begin{array}{lll}
r_{P} \cos \theta_{P i} & r_{P} \sin \theta_{P i} & h_{P}
\end{array}\right]^{T}, \\
\mathbf{b}_{i} & =\left[\begin{array}{lll}
r_{B} \cos \theta_{B i} & r_{B} \sin \theta_{B i} & h_{B}
\end{array}\right]^{T},
\end{aligned}
$$

where $T$ is the matrix transpose, $h_{P}$ is the $z$ coordinate of the upper universal joint of the $i$ th limb in the frame $O_{1}-X_{1} Y_{1} Z_{1}, h_{B}$ is the $z$ coordinate of the lower universal joint of the $i$ th limb in the frame $O-X Y Z$, and $\theta_{P i}$ and $\theta_{B i}$ are as follows:

$$
\begin{aligned}
& \theta_{P}=\left[\begin{array}{llllll}
\theta_{P 1} & \theta_{P 2} & \theta_{P 3} & \theta_{P 4} & \theta_{P 5} & \theta_{P 6}
\end{array}\right]^{T} \\
& =\left[\begin{array}{llllll}
\eta_{A} & \frac{2 \pi}{3}-\eta_{A} & \frac{2 \pi}{3}+\eta_{A} & \frac{4 \pi}{3}-\eta_{A} & \frac{4 \pi}{3}+\eta_{A} & -\eta_{A}
\end{array}\right]^{T}, \\
& \theta_{B}=\left[\begin{array}{llllll}
\theta_{B 1} & \theta_{B 2} & \theta_{B 3} & \theta_{B 4} & \theta_{B 5} & \theta_{B 6}
\end{array}\right]^{T} \\
& =\left[\begin{array}{llllll}
\eta_{B} & \frac{2 \pi}{3}-\eta_{B} & \frac{2 \pi}{3}+\eta_{B} & \frac{4 \pi}{3}-\eta_{B} & \frac{4 \pi}{3}+\eta_{B} & -\eta_{B}
\end{array}\right]^{T}
\end{aligned}
$$

where

$$
\begin{gathered}
\eta_{A}=\frac{\pi}{3}-\arcsin \left(\frac{d_{P}}{2 r_{P}}\right), \\
\eta_{B}=\arcsin \left(\frac{d_{B}}{2 r_{B}}\right) .
\end{gathered}
$$

For the $i$ th leg, the limb length $l_{i}$ was derived as

$$
l_{i}=l_{1 i}+l_{2 i}
$$

where $l_{i}$ is the length of $\overrightarrow{\mathrm{B}_{i}} \overrightarrow{\mathrm{P}}_{i}$.

Differentiating Equation (1), we obtained

$$
\delta \mathbf{p}+\delta \mathbf{R}^{\mathbf{L}} \mathbf{p}_{i}+\mathbf{R} \delta^{\mathbf{L}} \mathbf{p}_{i}=\delta \mathbf{b}_{i}+\delta l_{1 i} \mathbf{n}_{1 i}+l_{1 i} \delta \mathbf{n}_{1 i}+\delta l_{2 i} \mathbf{n}_{2 i}+l_{2 i} \delta \mathbf{n}_{2 i},
$$

where $\delta \mathbf{R}$ could be rewritten as follows, according to Reference [16]:

$$
\delta \mathbf{R}=\delta \boldsymbol{\theta} \times \mathbf{R}=\left[\begin{array}{ccc}
0 & -\delta \theta_{Z} & \delta \theta_{Y} \\
\delta \theta_{Z} & 0 & -\delta \theta_{X} \\
-\delta \theta_{Y} & \delta \theta_{X} & 0
\end{array}\right] \mathbf{R}=\mathbf{\Omega} \boldsymbol{R},
$$

where $\delta \boldsymbol{\theta}=\left[\begin{array}{lll}\delta \theta_{X} & \delta \theta_{Y} & \delta \theta_{Z}\end{array}\right]^{T}$ is the orientation error vector of the moving platform in the frame $O-X Y Z$, and $\delta \mathbf{p}=\left[\begin{array}{lll}\delta p_{X} & \delta p_{Y} & \delta p_{Z}\end{array}\right]^{T}$ is the translational error vector of the moving platform in the frame $O-X Y Z$.

Multiplication of $\mathbf{n}_{i}^{T}$ on both sides of Equation (10) yielded

$$
\begin{aligned}
& \mathbf{n}_{i}^{T} \delta \mathbf{p}+\mathbf{n}_{i}^{T} \delta \boldsymbol{\theta} \times \mathbf{R}^{\mathbf{L}} \mathbf{p}_{i}+\mathbf{n}_{i}^{T} \mathbf{R} \delta^{\mathbf{L}} \mathbf{p}_{i} \\
& =\mathbf{n}_{i}^{T} \delta \mathbf{b}_{i}+\mathbf{n}_{i}^{T} \delta l_{1 i} \mathbf{n}_{1 i}+\mathbf{n}_{i}^{T} l_{1 i} \delta \mathbf{n}_{1 i}+\mathbf{n}_{i}^{T} \delta l_{2 i} \mathbf{n}_{2 i}+\mathbf{n}_{i}^{T} l_{2 i} \delta \mathbf{n}_{2 i}
\end{aligned} .
$$


Since $\mathbf{n}_{i}^{T} \mathbf{n}_{i}=1, \mathbf{n}_{i}=\mathbf{n}_{1 i}=\mathbf{n}_{2 i}, \delta l_{2 i}=0$, and $\delta l_{i}=\delta l_{1 i}$, we had $\mathbf{n}_{i}^{T} \delta \mathbf{n}_{i}=0, \mathbf{n}_{i}^{T} \delta \mathbf{n}_{1 i}=0$, and $\mathbf{n}_{i}^{T} \delta \mathbf{n}_{2 i}=0$. Therefore, Equation (11) could be rewritten as

$$
\begin{aligned}
& \mathbf{n}_{i}^{T} \delta \mathbf{p}+\mathbf{n}_{i}^{T} \delta \boldsymbol{\theta} \times \mathbf{R}^{\mathbf{L}} \mathbf{p}_{i}+\mathbf{n}_{i}^{T} \mathbf{R} \delta^{\mathbf{L}} \mathbf{p}_{i} \\
& =\mathbf{n}_{i}^{T} \delta \mathbf{b}_{i}+\mathbf{n}_{i}^{T} \delta l_{1 i} \mathbf{n}_{i} \\
& =\mathbf{n}_{i}^{T} \delta \mathbf{b}_{i}+\delta l_{1 i} \\
& =\mathbf{n}_{i}^{T} \delta \mathbf{b}_{i}+\delta l_{i}
\end{aligned}
$$

$\delta l_{i}$ could be derived as

$$
\begin{aligned}
\delta l_{i}= & \mathbf{n}_{i}^{T} \delta \mathbf{p}+\mathbf{n}_{i}^{T} \delta \boldsymbol{\theta} \times \mathbf{R}^{\mathbf{L}} \mathbf{p}_{i}+\mathbf{n}_{i}^{T} \mathbf{R} \delta^{\mathbf{L}} \mathbf{p}_{i}-\mathbf{n}_{i}^{T} \delta \mathbf{b}_{i} \\
= & {\left[\begin{array}{ll}
\mathbf{n}_{i}^{T} & \left(\mathbf{R}^{\mathbf{L}} \mathbf{p}_{i} \times \mathbf{n}_{i}\right)^{T}
\end{array}\right]\left[\begin{array}{c}
\delta \mathbf{p} \\
\delta \boldsymbol{\theta}
\end{array}\right]+} \\
& {\left[\begin{array}{ll}
\mathbf{n}_{i}^{T} \mathbf{R} & \left(-\mathbf{n}_{i}^{T}\right)
\end{array}\right]\left[\begin{array}{c}
\delta^{\mathbf{L}} \mathbf{p}_{i} \\
\delta \mathbf{b}_{i}
\end{array}\right] }
\end{aligned}
$$

Once all six limbs were assembled, the errors could be expressed as

$$
\delta \mathbf{1}=\mathbf{J}_{P} \delta \mathbf{x}+\mathbf{J}_{s} \delta \mathbf{s}
$$

where

$$
\begin{aligned}
& \delta \mathbf{1}=\left[\begin{array}{lll}
\delta l_{1} & \cdots & \delta l_{6}
\end{array}\right]^{T} \in \Re^{6 \times 1}, \\
& \delta \mathbf{x}=\left[\begin{array}{c}
\delta \mathbf{p} \\
\delta \boldsymbol{\theta}
\end{array}\right] \in \Re^{6 \times 1}, \\
& \delta \mathbf{s}=\left[\begin{array}{c}
\delta^{\mathbf{L}} \mathbf{p}_{i} \\
\delta \mathbf{b}_{1} \\
\vdots \\
\delta^{\mathbf{L}} \mathbf{p}_{6} \\
\delta \mathbf{b}_{6}
\end{array}\right] \in \Re^{36 \times 1}, \\
& \mathbf{J}_{P}=\left[\begin{array}{cc}
\mathbf{n}_{1}^{T} & \left(\mathbf{R}^{\mathbf{L}} \mathbf{p}_{i} \times \mathbf{n}_{1}\right)^{T} \\
\vdots & \vdots \\
\mathbf{n}_{6}^{T} & \left(\mathbf{R}^{\mathbf{L}} \mathbf{p}_{6} \times \mathbf{n}_{6}\right)^{T}
\end{array}\right] \in \Re^{6 \times 6}, \\
& \mathbf{J}_{s}=\left[\begin{array}{ccccc}
\mathbf{n}_{1}^{T} \mathbf{R} & \left(-\mathbf{n}_{1}^{T}\right) & \cdots & \mathbf{0}_{1 \times 3} & \mathbf{0}_{1 \times 3} \\
\vdots & \vdots & \ddots & \vdots & \vdots \\
\mathbf{0}_{1 \times 3} & \mathbf{0}_{1 \times 3} & \cdots & \mathbf{n}_{6}^{T} \mathbf{R} & \left(-\mathbf{n}_{6}^{T}\right)
\end{array}\right] \in \Re^{6 \times 36}, \\
& \mathbf{0}_{1 \times 3}=\left[\begin{array}{lll}
0 & 0 & 0
\end{array}\right] \text {. }
\end{aligned}
$$

If the inverse of $\mathbf{J}_{P}$ existed, then $\delta \mathbf{x}$ could be derived as

$$
\delta \mathbf{x}=\mathbf{J}_{P}^{-1} \delta \mathbf{1}-\mathbf{J}_{P}^{-1} \mathbf{J}_{s} \delta \mathbf{s} .
$$

The first term on the right side represents the actuation-induced error, and the second term is the error caused by the position errors of the joints [16].

\section{Optimal Design Method}

In engineering, we may assume that the manufacturing tolerances on the geometrical parameters are bounded and the maximum values of $\delta \mathbf{1}$ and $\delta \mathbf{s}$ are known as a function of the manufacturing 
method. The maximum values of $\delta \mathbf{x}$ for the optimal design solutions had to be lower than the given accuracy by customers, before being dealt with as a constraint in the optimal design process in this paper.

Many scholars studied the optimal design of a GSP with only one optimal solution [24]. In practice, many different functional requirements of robots are intended to be satisfied [25]; thus, it is more appropriate to have multiple optimization solutions after the optimal kinematic design [24]. The condition number and determinant of the kinematic Jacobian matrix, $\mathbf{J}_{P}$, of a GSP were extensively used as optimal design objectives [24]; therefore, they were chosen as objective functions in the optimal design process in this paper. Accordingly, they were defined as

$$
\begin{gathered}
\operatorname{cond}\left(\mathbf{J}_{P}\right)=\frac{\sigma_{\max }\left(\mathbf{J}_{P}\right)}{\sigma_{\min }\left(\mathbf{J}_{P}\right)}, \\
\omega=\sqrt{\operatorname{det}\left(\mathbf{J}_{P} \mathbf{J}_{P}^{T}\right)}=\left|\operatorname{det}\left(\mathbf{J}_{P}\right)\right|,
\end{gathered}
$$

where $\sigma_{\max }\left(\mathbf{J}_{P}\right)$ and $\sigma_{\min }\left(\mathbf{J}_{P}\right)$ are the maximum and minimum singular values of $\mathbf{J}_{P}$ at one pose, respectively, and $\operatorname{det}\left(\mathbf{J}_{P}\right)$ is the determinant of $\mathbf{J}_{P}$.

In the optimal design process, evolutionary algorithms are extensively used to search the optimal solutions [26]. In this paper, the elitist non-dominated sorting genetic algorithm version II (NSGA-II), developed by Deb et al. [27], was employed to solve the multi-objective optimal design problems of the 6-UCU kind GSP, due to its good spread of solutions and convergence to obtain the Pareto front. Real-coded NSGA-II with a simulated binary crossover (SBX) operator [28] and a polynomial mutation operator [29] were adopted in this paper to search the minimum values of the objective functions. The parameters of the NSGA-II are shown in Table 1.

Table 1. Parameters of the non-dominated sorting genetic algorithm version II (NSGA-II).

\begin{tabular}{cc}
\hline Number of Iterations & $\mathbf{1 0 0 0}$ \\
\hline Population size & 50 \\
Crossover probability & 0.9 \\
Mutation probability & 0.1 \\
Distribution index for the simulated binary crossover (SBX) & 20 \\
Distribution index for the polynomial mutation & 20 \\
\hline
\end{tabular}

The optimal design procedures were proposed as described below.

Step 1. The customers usually provided the maximum motion requirements for each degree of freedom (DOF), as shown in Table 2.

Table 2. Typical requirements by customers.

\begin{tabular}{cccc}
\hline \multicolumn{3}{c}{ Payload } & \\
\hline Degree of Freedom (DOF) & Maximum Excursion & Speed & Acceleration \\
\hline Roll & $\pm R o_{X}\left({ }^{\circ}\right)$ & $\pm R v_{X}\left({ }^{\circ} / \mathrm{s}\right)$ & $\pm R a_{X}\left({ }^{\circ} / \mathrm{s}^{2}\right)$ \\
Pitch & $\pm R o_{Y}\left({ }^{\circ}\right)$ & $\pm R v_{Y}\left({ }^{\circ} / \mathrm{s}\right)$ & $\pm R a_{Y}\left({ }^{\circ} / \mathrm{s}^{2}\right)$ \\
Yaw & $\pm R o_{Z}\left({ }^{\circ}\right)$ & $\pm R v_{Z}\left({ }^{\circ} / \mathrm{s}\right)$ & $\pm R a_{Z}\left({ }^{\circ} / \mathrm{s}^{2}\right)$ \\
Surge & $\pm T r_{X}(\mathrm{~m})$ & $\pm T v_{X}(\mathrm{~m} / \mathrm{s})$ & $\pm T a_{X}\left(\mathrm{~m} / \mathrm{s}^{2}\right)$ \\
Sway & $\pm \operatorname{Tr}_{Y}(\mathrm{~m})$ & $\pm T v_{Y}(\mathrm{~m} / \mathrm{s})$ & $\pm T a_{Y}\left(\mathrm{~m} / \mathrm{s}^{2}\right)$ \\
Heave & $\pm \operatorname{Tr}_{Z}(\mathrm{~m})$ & $\pm T v_{Z}(\mathrm{~m} / \mathrm{s})$ & $\pm T a_{Z}\left(\mathrm{~m} / \mathrm{s}^{2}\right)$ \\
\hline
\end{tabular}


We transformed the requirements of the customer to 12 typical trajectories along six single axes [24]. Two typical travel trajectories of translation along the $X$ axis were

$$
\begin{gathered}
S_{X}(t)=T r_{X} \sin \left(\frac{T v_{X}}{T r_{X}} t\right), \\
S_{X}(t)=\frac{\left(T v_{X}\right)^{2}}{T a_{X}} \sin \left(\frac{T a_{X}}{T v_{X}} t\right),
\end{gathered}
$$

where $t$ is the run time.

Step 2. At each pose, $\operatorname{con} d\left(\mathbf{J}_{P}\right)$ was calculated using Equation (22). When it was at or near the singularity, calculating $\delta \mathbf{x}$ using Equation (21) was potentially wrong. We used another method to solve this problem, as follows: if $\operatorname{con} d\left(\mathbf{J}_{P}\right)>10^{6}$, then $\delta \mathbf{x}=\left[\begin{array}{llllll}10^{7} & 10^{7} & 10^{7} & 10^{7} & 10^{7} & 10^{7}\end{array}\right]^{T}$; if $\operatorname{con} d\left(\mathbf{J}_{P}\right) \leq 10^{6}, \delta \mathbf{x}$ was calculated using Equation (21). In the searching process, the guaranteed accuracy was given, and then it was dealt with as a constraint. The penalty function is the most often used technique in constrained optimization [26]; however, the penalty coefficients are very difficult to choose appropriately [30]. We handled the constraint as follows: if $\delta \mathbf{x}$ was in the required range, $\operatorname{con} d\left(\mathbf{J}_{P}\right)$ was calculated using Equation (22), and $\omega$ was calculated using Equation (23). Otherwise, $\operatorname{con} d\left(\mathbf{J}_{P}\right)=\left(10^{7}+f_{c}\right)$ and $\omega=\left(-f_{c}\right)$. A subprogram was subsequently created to calculate the maximum value of cond $\left(\mathbf{J}_{P}\right)$ and the minimum value of $\omega$ for all 12 typical trajectories.

$$
f_{c}=\left\|\frac{\delta p_{X}}{a c_{T X}}\right\|+\left\|\frac{\delta p_{Y}}{a c_{T Y}}\right\|+\left\|\frac{\delta p_{Z}}{a c_{T Z}}\right\|+\left\|\frac{\delta \theta_{X}}{a c_{R X}}\right\|+\left\|\frac{\delta \theta_{Y}}{a c_{R Y}}\right\|+\left\|\frac{\delta \theta_{Z}}{a c_{R Z}}\right\|,
$$

where $a c_{T X}, a c_{T Y}$, and $a c_{T Z}$ are the required maximum linear displacement positioning errors along the $X, Y$, and $Z$ axes, respectively; $a c_{R X}, a c_{R Y}$, and $a c_{R Z}$ are the required maximum angular displacement positioning errors along the $X, Y$, and $Z$ axes, respectively.

Step 3. cond $\left(\mathbf{J}_{P}\right)_{\max }$ was set as the maximum value of $\operatorname{con} d\left(\mathbf{J}_{P}\right)$ calculated in step 2 , and $\omega_{\min }$ was set as the minimum value of $\omega$ calculated in step 2 .

Step 4 . The design variables were chosen as $r_{P}, r_{B}, d_{P}, d_{B}$, and $H_{0}$, where $H_{0}$ was the height of the upper universal joint plane to the lower universal joint plane at the home position. $f_{1}=\operatorname{con} d\left(\mathbf{J}_{P}\right)_{\max }$ and $f_{2}=-\omega_{\min }$. NSGA-II was used to minimize the objective functions, $f_{1}$ and $f_{2}$, simultaneously.

For other requirements, the 12 typical trajectories were replaced by all the typical trajectories in the special applications. In the optimal kinematic design of a flight simulator, 31 typical trajectories of a Boeing 747 were considered in the design process by Advani [31]. If one desires a singularity-free workspace, as for motion simulators, the 12 typical trajectories in Step 2 would be replaced by all the typical trajectories and the 64 extreme positions [24,31].

\section{Case Study}

A case study is presented in this section to show the effectiveness of the proposed optimal design method. For this specific case, the requirements of the customer are shown in Table 3.

From a practical consideration, the ranges of the parameters were chosen as follows:

$h_{P}=-0.45(\mathrm{~m}), h_{B}=\left(-0.45-H_{0}\right)(\mathrm{m}), 0.75(\mathrm{~m}) \leq r_{B} \leq 3.0(\mathrm{~m}), 0.75(\mathrm{~m}) \leq r_{P} \leq 3.0(\mathrm{~m})$, $r_{P} \leq r_{B}, 1.0(\mathrm{~m}) \leq H_{0} \leq 3.5(\mathrm{~m}), 0.25(\mathrm{~m}) \leq d_{B} \leq r_{B}$, and $0.22(\mathrm{~m}) \leq d_{P} \leq r_{P}$. The range of every element of $\delta 1$ and $\delta \mathbf{s}$ were in the range $[-0.3,0.3](\mathrm{mm})$. 
Table 3. Requirements of the customer.

\begin{tabular}{|c|c|c|c|}
\hline \multicolumn{2}{|r|}{ Payload } & \multicolumn{2}{|c|}{$10,000 \mathrm{~kg}$} \\
\hline \multirow{2}{*}{\multicolumn{2}{|c|}{ Maximum Position Errors }} & Linear Travel & $1 \mathrm{~mm}$ \\
\hline & & Angular Travel & $0.1^{\circ}$ \\
\hline DOF & Maximum Excursion & Speed & Acceleration \\
\hline Roll & $\pm 25^{\circ}$ & $\pm 20^{\circ} / \mathrm{s}$ & $\pm 210^{\circ} / \mathrm{s}^{2}$ \\
\hline Pitch & $\pm 25^{\circ}$ & $\pm 20^{\circ} / \mathrm{s}$ & $\pm 210^{\circ} / \mathrm{s}^{2}$ \\
\hline Yaw & $\pm 30^{\circ}$ & $\pm 20^{\circ} / \mathrm{s}$ & $\pm 210^{\circ} / \mathrm{s}^{2}$ \\
\hline Surge & $\pm 1 \mathrm{~m}$ & $\pm 0.7 \mathrm{~m} / \mathrm{s}$ & $\pm 10 \mathrm{~m} / \mathrm{s}^{2}$ \\
\hline Sway & $\pm 1 \mathrm{~m}$ & $\pm 0.7 \mathrm{~m} / \mathrm{s}$ & $\pm 10 \mathrm{~m} / \mathrm{s}^{2}$ \\
\hline Heave & $\pm 0.8 \mathrm{~m}$ & $\pm 0.6 \mathrm{~m} / \mathrm{s}$ & $\pm 10 \mathrm{~m} / \mathrm{s}^{2}$ \\
\hline
\end{tabular}

After applying the proposed optimization process, as outlined in Section 3, 50 optimal solutions and the Pareto-optimal front were found, as shown in Figure 4. Lastly, there were three optimum design points, labeled as a, b, and c, whose corresponding objective values and design parameters are shown in Table 4. As seen in Table 4, the maximum errors of all the linear displacements were lower than $1 \mathrm{~mm}$, and the maximum errors of all the angular displacements were lower than $0.1^{\circ}$. Therefore, all the requirements by the customer, given in Table 3, were met. This showed the effectiveness of the proposed method for the optimal design purpose.

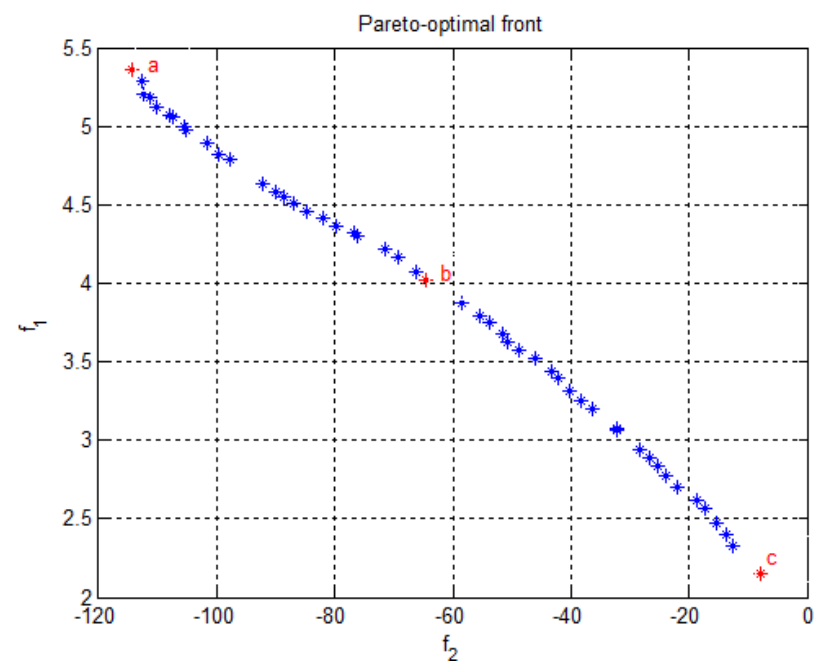

Figure 4. Pareto-optimal front of the solutions.

Table 4. Objective functions values from the Pareto sets and their corresponding design variables.

\begin{tabular}{cccc}
\hline & $\mathbf{a}$ & $\mathbf{b}$ & $\mathbf{c}$ \\
\hline$d_{B}(\mathrm{~m})$ & 0.3409 & 0.3489 & 0.2500 \\
$d_{P}(\mathrm{~m})$ & 1.6915 & 0.5177 & 0.2200 \\
$r_{B}(\mathrm{~m})$ & 3.0000 & 2.9981 & 2.6552 \\
$r_{P}(\mathrm{~m})$ & 3.0000 & 2.4046 & 1.2128 \\
$H_{0}(\mathrm{~m})$ & 2.4002 & 2.4044 & 2.4363 \\
$f_{2}$ & -114.2709 & -64.5399 & -8.0017 \\
$f_{1}$ & 5.3615 & 4.0201 & 2.1464 \\
$\delta p_{X}(\mathrm{~mm})$ & 0.2 & 0.2 & 0.2 \\
$\delta p_{Y}(\mathrm{~mm})$ & 0.2 & 0.2 & 0.2 \\
$\delta p_{Z}(\mathrm{~mm})$ & 0.6 & 0.6 & 0.6 \\
$\delta \theta_{X}\left({ }^{\circ}\right)$ & 0.0022 & 0.0025 & 0.0020 \\
$\delta \theta_{Y}\left({ }^{\circ}\right)$ & 0.0018 & 0.0027 & 0.0026 \\
$\delta \theta_{Z}\left({ }^{\circ}\right)$ & 0.0030 & 0.0029 & 0.0030 \\
\hline
\end{tabular}




\section{Conclusions}

Motion simulators extensively use 6-UCU kind GSPs. A differential error model for a 6-UCU kind GSP was derived in this paper, and contained both the actuation-induced error and the error caused by the position errors of the joints. The guaranteed given accuracy was used as a constraint in the optimal design process of the high-accuracy motion simulators. An optimal kinematic design method was proposed by using the multi-objective evolutionary algorithm, NSGA-II. If $\mathbf{J}_{P}$ was near or at the singularity pose, the inverse of $\mathbf{J}_{P}$ potentially did not exist. $\delta \mathbf{x}$ could be wrongly calculated using Equation (21). In order to solve this problem, a method was presented where the condition number of $\mathbf{J}_{P}$ was compared with a large number. Another engineering method was proposed to handle the accuracy requirement constraints in the optimal design process. Multiple optimization solutions were found following implementation of the optimal kinematic design.

The effectiveness of the proposed method was verified through a practical optimal design case. The proposed optimal design method can be used as a guideline for the practical design of GSPs used in other applications with high-accuracy requirements.

Conflicts of Interest: The authors declare no conflict of interest.

\section{References}

1. Merlet, J.P. Parallel Robots, 2nd ed.; Springer: Dordrecht, The Netherlands, 2006; pp. 77-78. ISBN 978-1-4020-4132-7.

2. Huang, Z.; Kong, L.F.; Fang, Y.F. Mechanism Theory of Parallel Robotic Manipulator and Control; China Mechanical Press: Beijing, China, 1997; pp. 306-308. ISBN 7-111-05812-7. (In Chinese)

3. Fichter, E.; Kerr, D.; Rees-Jones, J. The Gough-Stewart platform parallel manipulator: A retrospective appreciation. Proc. Inst. Mech. Eng. Part C J. Mech. Eng. Sci. 2009, 223, 243-281. [CrossRef]

4. Gough, V.E.; Whitehall, S.G. Universal Tyre Testing Machine. In Proceedings of the 9th International Automobile Technical Congress, Bursa, Turkey, 7-8 May 2018.

5. Stewart, D. A Platform with six degrees of freedom. Proc. Inst. Mech. Eng. 1965, 180, 371-386. [CrossRef]

6. Cappel, K.L. Motion Simulator. U.S. Patent 3,295,224, 3 January 1967.

7. Ma, O. Mechanical Analysis of Parallel Manipulators with Simulation, Design and Control Applications. Ph.D. Thesis, McGill University, Montreal, QC, Canada, 1991.

8. Cerda Salzmann, D.J. Ampelmann: Development of the Access System for Offshore Wind Turbines. Ph.D. Thesis, Delft University of Technology, Delft, The Netherlands, 2010.

9. Blaise, J.; Bonev, I.; Monsarrat, B.; Briot, S.; Lambert, J.M.; Perron, C. Kinematic characterisation of hexapods for industry. Ind. Robot Int. J. 2010, 37, 79-88. [CrossRef]

10. VARIAX: The Machine Tool of the Future-Today! Giddings \& Lewis, Inc.: Fond Du Lac, WI, USA, 1994.

11. Koch, P.M.; Kesteven, M.; Nishioka, H.; Jiang, H.; Lin, K.Y.; Umetsu, K.; Huang, Y.-D.; Raffin, P.; Chen, K.J.; Ibañez-Romano, F. The AMiBA hexapod telescope mount. Astrophys. J. 2009, 694, 1670-1684. [CrossRef]

12. Liu, G.; Zheng, S.; Ogbobe, P.; Han, J. Inverse kinematic and dynamic analyses of the 6-UCU parallel manipulator. Appl. Mech. Mater. 2012, 127, 172-180. [CrossRef]

13. Liu, G.; Qu, Z.; Liu, X.; Han, J. Singularity analysis and detection of 6-UCU parallel manipulator. Robot Comput. Integr. Manuf. 2014, 30, 172-179. [CrossRef]

14. Dai, X.; Huang, Q.; Han, J.; Li, H. Accuracy Synthesis of Stewart Platform used in Testing System for Spacecraft Docking Mechanism. In Proceedings of the International Conference on Measuring Technology and Mechatronics Automation, ICMTMA'09, Zhangjiajie, China, 11-12 April 2009; Volume 3, pp. 7-10. [CrossRef]

15. Wang, J.; Masory, O. On the Accuracy of a Stewart Platform-Part I: The Effect of Manufacturing Tolerances. In Proceedings of the IEEE International Conference on Robotics and Automation, Atlanta, GA, USA, 2-6 May 1993; pp. 114-120. [CrossRef]

16. Ropponen, T.; Arai, T. Accuracy Analysis of a Modified Stewart Platform Manipulator. In Proceedings of the IEEE International Conference on Robotics and Automation, Nagoya, Japan, 21-27 May 1995; pp. 521-525. [CrossRef] 
17. Patel, A.J.; Ehmann, K.F. Volumetric error analysis of a Stewart platform-based machine tool. CIRP Ann. Manuf. Technol. 1997, 46, 287-290. [CrossRef]

18. Wang, S.M.; Ehmann, K.F. Error model and accuracy analysis of a six-DOF Stewart platform. J. Manuf. Sci. Eng. 2002, 124, 286-295. [CrossRef]

19. Masory, O.; Wang, J.; Zhuang, H. On the Accuracy of a Stewart Platform-Part II: Kinematic Calibration and Compensation. In Proceedings of the IEEE International Conference on Robotics and Automation, Atlanta, GA, USA, 2-6 May 1993; pp. 725-731. [CrossRef]

20. Cong, D.; Yu, D.; Han, J. Kinematics accuracy analysis and error compensation of Stewart platform. J. Eng. Des. 2006, 13, 162-165. (In Chinese)

21. Merlet, J.P.; Daney, D. Dimensional Synthesis of Parallel Robots with a Guaranteed Given Accuracy over a Specific Workspace. In Proceedings of the IEEE International Conference on Robotics and Automation, ICRA 2005, Barcelona, Spain, 18-22 April 2005; pp. 942-947. [CrossRef]

22. Merlet, J.-P.; Daney, D. Appropriate Design of Parallel Manipulators. In Smart Devices and Machines for Advanced Manufacturing; Wang, L., Xi, F., Eds.; Springer: London, UK, 2008; pp. 1-25. ISBN 978-1-84800-146-6.

23. Merlet, J.P. Interval analysis and reliability in robotics. Int. J. Reliab. Saf. 2009, 3, 104-130. [CrossRef]

24. Liu, G.; Qu, Z.; Han, J.; Liu, X. Systematic optimal design procedures for the Gough-Stewart platform used as motion simulators. Ind. Robot 2013, 40, 550-558. [CrossRef]

25. Angeles, J.; Park, F.C. Design and performance evaluation. In Springer Handbook of Robotics, 2nd ed.; Siciliano, B., Khatib, O., Eds.; Springer: Berlin, Germany, 2016; pp. 399-418. ISBN 978-3-319-32550-7.

26. Yu, X.; Gen, M. Introduction to Evolutionary Algorithms; Springer: London, UK, 2010; pp. 3-8. ISBN 978-1-84996-128-8.

27. Deb, K.; Pratap, A.; Agarwal, S.; Meyarivan, T.A.M.T. A fast and elitist multiobjective genetic algorithm: NSGA-II. IEEE Trans. Evolut. Comput. 2002, 6, 182-197. [CrossRef]

28. Deb, K.; Agrawal, R.B. Simulated binary crossover for continuous search space. Complex Syst. 1994, 9, 1-34.

29. Deb, K.; Goyal, M. A combined genetic adaptive search (GeneAS) for engineering design. Comput. Sci. Inf. Syst. 1996, 26, 30-45.

30. Deb, K. An efficient constraint handling method for genetic algorithms. Comput. Method Appl. Mech. 2000, 186, 311-338. [CrossRef]

31. Advani, S.K. The Kinematic Design of Flight Simulator Motion Bases. PhD. Dissertation, TU Delft, Delft, The Netherlands, 1998; pp. 103-191. 